\title{
HILBERT INTEGRAL OPERATOR INEQUALITIES
}

\author{
KUANG JiCHANG AND THEMISTOCles M. RASSIAS
}

Abstract. In this paper, we establish new Hilbert integral operator inequalities with the general kernel. They are significant extensions and improvements of some known results.

Mathematics subject classification (1991): 26D10.

Key words and phrases: Hilbert inequality, integral operator, kernel.

\section{REFERENCES}

[1] G. H. Hardy, J. E. LitTlewood AND G. Polya, Inequalities, Cambridge University Press, Cambridge, MA, 1952.

[2] A. E. INGHAM, A note on Hilbert's inequality, J. London Math. Soc. 11 (1936), 237-240.

[3] YANG Bicheng, On Hilbert's integral inequality, J. Math. Anal. Appl. 220 (1998), 778-785.

[4] Gao Mingzhe, Tan Li And L. Debnath, Some improvements on Hilbert's integral inequality, J. Math. Anal. Appl. 229 (1999), 682-689.

[5] Kuang Jichang, On Hilbert's integral inequality, J. Math. Anal. Appl. 233 (1999).

[6] YANG Bicheng, On generalizations of Hardy-Hilbert's integral inequalities, Acta Math. Sinica 41 No. 4 (1998), 839-844. 